\title{
The Steiner Problem for Count Matroids
}

\author{
Tibor Jordán ${ }^{1(\otimes)}$, Yusuke Kobayashi² ${ }^{2}$ Ryoga Mahara ${ }^{2}$, \\ and Kazuhisa Makino ${ }^{2}$
}

1 Department of Operations Research, Eötvös University, and the MTA-ELTE Egerváry Research Group on Combinatorial Optimization, Pázmány Péter sétány 1/C, Budapest 1117, Hungary

jordan@cs.elte.hu

2 Research Institute for Mathematical Sciences, Kyoto University, Kyoto 606-8502, Japan

\{yusuke, ryoga, makino\}@kurims . kyoto-u.ac.jp

\begin{abstract}
We introduce and study a generalization of the well-known Steiner tree problem to count matroids. In the count matroid $\mathcal{M}_{k, l}(G)$, defined on the edge set of a graph $G=(V, E)$, a set $F \subseteq E$ is independent if every vertex set $X \subseteq V$ spans at most $k|X|-l$ edges of $F$. The graph is called $(k, l)$-tight if its edge set is independent in $\mathcal{M}_{k, l}(G)$ and $|E|=k|V|-l$ holds.

Given a graph $G=(V, E)$, a non-negative length function $w: E \rightarrow \mathbb{R}$, a set $T \subseteq V$ of terminals and parameters $k, l$, our goal is to find a shortest $(k, l)$-tight subgraph of $G$ that contains the terminals. Since $\mathcal{M}_{1,1}(G)$ is isomorphic to the graphic matroid of $G$, the special case $k=l=1$ corresponds to the Steiner tree problem. We obtain other interesting problems by choosing different parameters: for example, in the case $k=2, l=3$ the target is a shortest rigid subgraph containing all terminals.

First we show that this problem is NP-hard even if $k=2, l=3$, and $w$ is metric, or $w \equiv 1$ and $|T|=2$. As a by-product of this result we obtain that finding a shortest circuit in $\mathcal{M}_{2,3}(G)$ is NP-hard.

Then we design a $(k+1)$-approximation algorithm for the metric version of the problem with parameters $(k, k+1)$, for all $k \geq 2$. In particular, we obtain a 3-approximation algorithm for the Steiner version of the shortest rigid subgraph problem. We also show that the metric version can be solved in polynomial time for $k=2, l=3$, provided $|T|$ is fixed.
\end{abstract}

Keywords: Count matroid $\cdot$ Steiner problem $\cdot$ Rigid graph

This work was supported by the Research Institute for Mathematical Sciences, an International Joint Usage/Research Center located in Kyoto University, the JSPS KAKENHI grant no. JP18H05291, and the Hungarian Scientific Research Fund grant no. K 109240. The first author was also supported by Project ED-18-1-2019-030 (Applicationspecific highly reliable IT solutions), which has been implemented with the support provided from the National Research, Development and Innovation Fund of Hungary, financed under the Thematic Excellence Programme funding scheme.

(C) Springer Nature Switzerland AG 2020

L. Gąsieniec et al. (Eds.): IWOCA 2020, LNCS 12126, pp. 330-342, 2020.

https://doi.org/10.1007/978-3-030-48966-3_25 


\section{Introduction}

Let $k$ be a positive integer and let $l$ be an integer satisfying $2 k-l \geq 1$. We say that a graph $G=(V, E)$ is $(k, l)$-sparse if

$$
i_{G}(X) \leq k|X|-l, \text { for all } X \subseteq V \text { with }|X| \geq 2,
$$

where $i_{G}(X)$ denotes the number of edges induced by $X$ in $G$. The graph is called $(k, l)$-tight if it is $(k, l)$-sparse and $|E|=k|V|-l$ holds. It is well-known that the edge sets of the $(k, l)$-sparse subgraphs of a graph $G$ form the independent sets of a matroid, defined on the edge set of $G$. This matroid, denoted by $\mathcal{M}_{k, l}(G)$, is called the count matroid of $G$, with parameters $k, l$, see e.g. [5,17].

For a graph $G=(V, E)$ and set $T \subseteq V$ of terminal vertices, we say that a subgraph $H=\left(V^{\prime}, E^{\prime}\right)$ of $G$ is $T$-( $(k, l)$-tight if $H$ is $(k, l)$-tight and $T \subseteq V^{\prime}$. Given a graph $G=(V, E)$, a terminal set $T \subseteq V$, a length function $w: E \rightarrow \mathbb{R}_{+}$, and parameters $k, l$, the shortest $T$ - $(k, l)$-tight subgraph problem is to find a $T$ $(k, l)$-tight subgraph $H$ of $G$ with minimum total edge-length. If $G$ is a complete graph and $w$ is metric (that is, $w$ satisfies the triangle inequality), this problem is called the metric shortest $T-(k, l)$-tight subgraph problem. Note that we use $\mathbb{R}_{+}$to denote the set of non-negative real numbers.

Since $\mathcal{M}_{1,1}(G)$ is isomorphic to the graphic matroid of $G$, the special case $k=l=1$ corresponds to the Steiner tree problem. Although we may obtain other interesting optimization problems by choosing different parameters (see below), this is the only special case of our general problem - that we call the Steiner problem for count matroids - that has been studied before.

\subsection{Previous Work}

The Steiner tree problem is one of the fundamental problems in combinatorial optimization: given a graph $G=(V, E)$, a terminal set $T \subseteq V$, a length function $w: E \rightarrow \mathbb{R}_{+}$, find a shortest tree in $G$ which contains all terminal vertices. It is NP-hard. It is known that there is an approximation factor preserving reduction to its metric version. The best known approximation factor, due to Byrka et al. $[2]$, is 1.39. It is also well-known that it can be solved in polynomial time if $|T|=2$ (which is a shortest path problem) and more generally, if $|T|$ is fixed. This problem has numerous other versions and extensions, see e.g. [3,4].

A related notion, which is also relevant in the context of count matroids, is the Steiner ratio. Consider a metric instance of a Steiner problem, in which we have a complete graph $G=(V, E)$, a terminal set $T \subseteq V$, and a length function $w: E \rightarrow \mathbb{R}_{+}$, and we wish to find a shortest subgraph $H$ of $G$ that contains all terminals and satisfies a given property. For example, we may want to find a connected subgraph, but we can also think of other properties (e.g. $k$-edge-connected or $(k, l)$-tight) satisfied by $G[T]$ (i.e. the complete subgraph of $G$ induced by $T$ ).

Then the total length of an optimal solution divided by the length of a shortest spanning subgraph of $G[T]$ that satisfies the given property is called 
the Steiner ratio of the instance. The Steiner ratio of the (metric) problem is the best possible lower bound on the Steiner ratio that is valid for all instances.

Note that, just like in the Steiner tree problem, the shortest $(k, l)$-tight spanning subgraph of $G[T]$, if it exists, can be found in polynomial time by a greedy algorithm. It holds for all parameters $k, l$, due to the matroidal nature of the problem, see e.g. [5].

\subsection{Motivation and New Results}

Our motivation to introduce and study this problem comes from rigidity theory and its applications. In this area count matroids play an important role. For example, a graph (realized as a generic two-dimensional bar-and-joint structure) is rigid if and only if it contains a $(2,3)$-tight spanning subgraph (see Sect.2). Thus, by choosing $k=2$ and $l=3$ in our problem, we look for the shortest rigid subgraph of a graph that contains a designated set of vertices. Other well-studied parameters that show up in e.g. parallel drawing and in rigidity problems of body-bar and body-hinge frameworks include the cases when $l=k$ and $l=k+1$, for all $k \geq 2$. See [17] for more on these connections. Approximation algorithms for these counts may also be useful in variants of the sensor network localization problem, where rigidity theory plays a key role, see [7].

Another reason for investigating the complexity of the Steiner problem for count matroids is to have a better understanding of the problem of finding the girth of a (count) matroid, see $[14,15]$. We shall see that the problem of finding a shortest circuit containing a given element in a matroid $\mathcal{M}_{2,3}(G)$ is equivalent to the corresponding Steiner problem with two terminals.

We first show that the Steiner problem for count matroids is NP-hard, even if $k=2, l=3$, and $w$ is metric, or $w \equiv 1$ and $|T|=2$. The latter result settles the complexity status of the girth problem for count matroids with parameters $k=2, l=3$. It also illustrates that - apart from the graphic matroid (the Steiner tree problem) and the bicircular matroid (see Sect.6) - the Steiner problem for count matroids is hard even for two terminals.

Then we give a $(k+1)$-approximation algorithm for the metric version for the counts $(k, k+1)$, for all $k \geq 2$. This specializes to a 3 -approximation algorithm for the shortest rigid subgraph problem. As a corollary we obtain that the Steiner ratio of the metric shortest $T$ - $(k, k+1)$-tight subgraph problem is between $\frac{1}{2}$ and $\frac{1}{k+1}$.

We also show that the (metric) shortest $T$-(2,3)-tight subgraph problem can be solved in polynomial time for fixed $|T|$. The algorithm is based on a structural result: we prove that there always exists an optimal solution $H$ with $|V(H)| \leq$ $15|T|-1$. It shows that, unlike in the case of the Steiner tree problem, the behaviour of the metric version is quite different from that of the case of general length functions. It is another new phenomenon for general counts.

We have similar results for the shortest $T$ - $(k, k)$-tight subgraph problem for all $k \geq 2$. By a result of Nash-Williams (see Theorem 3 below) a graph is $(k, k)$ tight if and only if its edge set can be decomposed into $k$ disjoint spanning trees. Although these graphs are well-studied and occur in important applications, 
we omit the results on $(k, k)$-tight subgraphs from this extended abstract: the $(k, k+1)$-tight case appears to be more involved and the methods used are similar.

\section{Preliminary Results}

In this section we make some preliminary observations and introduce some notions and earlier results we shall use in this paper.

\subsection{The Extension Operation}

We shall use the following operation on graphs several times. Let $G=(V, E)$ be a simple graph. The $(k, i)$-extension operation, for some integers $k \geq 1$ and $0 \leq i \leq k$, removes $i$ edges $u_{1} v_{1}, u_{2} v_{2}, \ldots u_{i} v_{i} \in E$ from $G$, and adds a new vertex $r$ and new edges $r u_{1}, \ldots, r u_{i}, r v_{1}, \ldots, r v_{i}, r w_{1}, \ldots, r w_{k-i}$, for some vertices $w_{1}, \ldots, w_{k-i}$ of $G$, in such a way that the resulting graph $G^{\prime}$ remains simple. Notice that the new vertex $r$ has degree $k+i$ in $G^{\prime}$.

The following lemma (which is implicit in [6]) is easy to verify. We remark that the lemma - with minor changes - holds for multigraphs, too. In this paper we restrict ourselves to simple graphs.

Lemma 1. Let $G=(V, E)$ be a $(k, k+1)$-tight simple graph and suppose that $G^{\prime}$ is obtained from $G$ by $a(k, i)$-extension operation for some $0 \leq i \leq k$. Then $G^{\prime}$ is also $(k, k+1)$-tight.

As the first application of Lemma 1 we show that for every $t \geq 2 k+1$ there exist $(k, k+1)$-tight graphs on $t$ vertices.

Lemma 2. Let $k$ and $t$ be integers with $k \geq 2$ and $t \geq 2 k+1$. Define $C_{t, k}$ as the graph whose vertex set and edge set are $\left\{x_{1}, \ldots, x_{t}\right\}$ and $\left\{x_{i} x_{i+1}, x_{i} x_{i+2}, \ldots\right.$, $\left.x_{i} x_{i+k} \mid i \in\{1, \ldots, t\}\right\}$, respectively, where we denote $x_{t+j}=x_{j}$ for $j=1, \ldots, t$. Let $C_{t, k}^{\prime}:=C_{t, k}-\left\{x_{1} x_{t}, x_{1} x_{t-1}, \ldots, x_{1} x_{t-k+1}, x_{k} x_{t}\right\}$. Then, $C_{t, k}^{\prime}$ is a $(k, k+1)$ tight graph.

Proof. We show that $C_{t, k}^{\prime}$ is a $(k, k+1)$-tight graph by induction on $t$. We first consider the case of $t=2 k+1$. Let $K_{2 k}$ be the complete graph with $2 k$ vertices $x_{2}, x_{3}, \ldots, x_{2 k}, x_{2 k+1}$. Then, $K_{2 k}-\left\{x_{k} x_{2 k+1}\right\}$ is a $(k, k+1)$-tight graph by a simple counting argument. Since $C_{2 k+1, k}^{\prime}$ is obtained from $K_{2 k}-\left\{x_{k} x_{2 k+1}\right\}$ by a $(k, 0)$-extension operation (which adds a new vertex $x_{1}$ and $k$ new edges $\left.x_{1} x_{2}, x_{1} x_{3}, \ldots, x_{1} x_{k}, x_{1} x_{k+1}\right)$, we have that $C_{2 k+1, k}^{\prime}$ is a $(k, k+1)$-tight graph by Lemma 1 . This shows the base case of the induction.

To show the induction step, assume that $C_{t, k}^{\prime}$ is a $(k, k+1)$-tight graph. We observe that $x_{i} x_{t-k+i} \in E\left(C_{t, k}^{\prime}\right)$ for $i=2,3, \ldots, k-1$. Since $C_{t+1, k}^{\prime}$ is obtained from $C_{t, k}^{\prime}$ by a $(k, k-2)$-extension operation (which adds a new vertex $x_{t+1}$ together with $2 k-2$ new edges $x_{t+1} x_{i}$ for $i=2,3, \ldots, k-1, t-k, t-k+1, \ldots, t-1$ and removes $x_{i} x_{t-k+i}$ for $\left.i=2,3, \ldots, k-1\right)$, we have that $C_{t+1, k}^{\prime}$ is a $(k, k+1)$ tight graph by Lemma 1 . This completes the proof. 


\subsection{Rigid Graphs}

We say, somewhat informally, that a graph $G=(V, E)$ is generically rigid in the plane if every bar-and-joint framework in the plane with underlying graph $G$ and with generic vertex coordinates is rigid: that is, every continuous motion of the vertices in the plane that preserves the edge lengths preserves all pairwise distances. Laman [12] proved that $G$ is generally rigid if and only if it has a (2,3)-tight spanning subgraph (or equivalently, its rigidity matroid $\mathcal{M}_{2,3}(G)$ has rank $2|V|-3)$. See [17] for an introduction to rigidity theory and for further count parameters that show up in this field, and [9] for more details on the combinatorial and matroidal aspects of two-dimensional rigidity.

Thus the Steiner problem for count matroids contains the problem of finding a shortest rigid subgraph containing a given a set of terminals. Since we shall mostly focus on this special case, for simplicity we shall also use $T$-rigid instead of saying that a subgraph which has a $T$-(2,3)-tight spanning subgraph. In this context minimally $T$-rigid corresponds to $T$ - $(2,3)$-tight.

The extension operations with parameters $(2,0)$ and $(2,1)$ introduced above play an important role in rigidity theory. If the parameter $k=2$ is clear from the context we use 0 -extension and 1-extension to mean a $(2,0)$ - or $(2,1)$-extension operation, respectively.

Lemma 3 [9]. Let $G=(V, E)$ be a minimally rigid graph and suppose that $G^{\prime}$ is obtained from $G$ by a 0 -extension or a 1-extension operation. Then $G^{\prime}$ is minimally rigid.

\subsection{Feasibility, Components, and Sparse Input Graphs}

In this subsection we consider $(2,3)$-sparsity (and rigidity), but the results easily extend to all counts studied in this paper.

A basic question concerning an instance of the Steiner problem for count matroids is whether there exists a feasible solution. The answer is based on the concept of rigid components: a rigid component of a graph $G$ is a maximal rigid subgraph. It is known that two rigid components have at most one vertex in common and that the family of rigid components can be found in polynomial time [9]. Since $|T| \geq 2$, it follows that all feasible solutions, if they exist, are subgraphs of the same rigid component of $G$. Furthermore, there is a feasible solution if and only if $G$ has a rigid component which contains all the terminals. In this case we can simply delete the complement of this rigid component and assume that the input graph is rigid.

Next suppose that the input graph $G=(V, E)$ is minimally rigid, that is, rigid and sparse at the same time. A useful observation is that the shortest $T$ rigid subgraph problem has a simple and efficient solution in this case. It follows from the next lemma, see e.g. [9].

Lemma 4. Let $G=(V, E)$ be a minimally rigid graph and let $G_{1}, G_{2}$ be minimally rigid subgraphs of $G$ with $\left|V\left(G_{1}\right) \cap V\left(G_{2}\right)\right| \geq 2$. Then $G_{1} \cap G_{2}$ is minimally rigid. 
Thus there is a unique smallest rigid subgraph of $G$ that contains $T$. Since $w$ is non-negative, it is an optimal solution.

The following result shows that we can find this smallest rigid subgraph efficiently. For a given $S \subseteq V$ with $|S| \geq 2$ let $C_{S}(G)$ be the unique smallest rigid subgraph of $G$ with $S \subseteq V\left(C_{S}(G)\right)$. If $S=\{a, b\}$ then we also use the notation $C_{a, b}(G)$.

Lemma 5 [10]. Let $G=(V, E)$ be a minimally rigid graph and $S \subseteq V$ with $|S| \geq 2$. Then

$$
C_{S}(G)=\bigcup_{a, b \in S} C_{a, b}(G)
$$

Lemma 5 shows that we can compute $C_{S}(G)$ by computing $C_{a, b}(G)$ for all pairs in $S$. It is not hard to see that for a given pair $a, b \in S$ the (edge set of) $C_{a, b}(G)$ is either $a b$ (if $a$ and $b$ are adjacent) or it is equal to the fundamental circuit of $a b$ with respect to $E$ (which is a base in the count matroid $\mathcal{M}_{2,3}(G)$ ). Since we have polynomial time independence oracles (using network flows, bipartite matchings, or graph orientations $[1,13])$, we can find all $C_{a, b}(G)$ 's and $C_{S}(G)$ in polynomial time.

Finally, consider the case when $p:=|E|-(2|V|-3)$ is a fixed constant for the input graph $G=(V, E)$. Let $m=|E|$. Then $G$ has at most $\left(\begin{array}{c}m \\ p\end{array}\right)$ minimally rigid spanning subgraphs.

Since every (minimally rigid) feasible solution can be extended to a minimally rigid spanning subgraph of $G$, and there is a unique smallest optimal solution whenever the input is minimally rigid, we can find an optimal solution by enumerating all minimally rigid spanning subgraphs of $G$ and computing the unique smallest rigid subgraph containing $T$ in each of them.

Proposition 1. The shortest T-rigid subgraph problem is polynomial time solvable if $p:=|E|-(2|V|-3)$ is a fixed constant for the input graph $G=(V, E)$.

\section{Hardness Results}

The proof of the next lemma is given in the full version of the paper [11].

Lemma 6. The shortest T-rigid subgraph problem is NP-hard even if $w(e)=1$ for every $e \in E$.

We can strengthen Lemma 6 as follows.

Theorem 1. The shortest T-rigid subgraph problem is NP-hard even if $w(e)=1$ for every $e \in E$ and $|T|=2$.

Proof. Lemma 6 shows that the shortest $T$-rigid subgraph problem is NP-hard even if $w(e)=1$ for every $e \in E$. We reduce this problem to the case of $|T|=2$.

Let $G=(V, E)$ and $T \subseteq V$ be an instance of the shortest $T$-rigid subgraph problem such that $|T| \geq 3$ and $w(e)=1$ for every $e \in E$. Pick up two distinct 
terminals $t_{1}, t_{2} \in T$ arbitrarily. Construct a new graph $G^{\prime}=\left(V^{\prime}, E^{\prime}\right)$ from $G$ by adding a new vertex $v$ together with two edges $v t_{1}$ and $v t_{2}$. Let $T^{\prime}=$ $\left(T \backslash\left\{t_{1}, t_{2}\right\}\right) \cup\{v\}$. Then, the obtained instance $\left(G^{\prime}, T^{\prime}\right)$ is equivalent to the original instance $(G, T)$ in the following sense. If $G$ contains a $T$-rigid subgraph $H=\left(V_{H}, E_{H}\right)$ with $k$ edges, then $H^{\prime}=\left(V_{H} \cup\{v\}, E_{H} \cup\left\{v t_{1}, v t_{2}\right\}\right)$ is a $T^{\prime}$-rigid subgraph of $G^{\prime}$ with $k+2$ edges. Conversely, if $G^{\prime}$ contains a $T^{\prime}$-rigid subgraph $H^{\prime}=\left(V_{H^{\prime}}, E_{H^{\prime}}\right)$ with $k+2$ edges, then $H=\left(V_{H^{\prime}} \backslash\{v\}, E_{H^{\prime}} \backslash\left\{v t_{1}, v t_{2}\right\}\right)$ is a $T$-rigid subgraph of $G$ with $k$ edges by Lemma 3 .

By repeating this procedure $|T|-2$ times, we obtain a graph $G^{*}=\left(V^{*}, E^{*}\right)$ and $T^{*} \subseteq V^{*}$ with $\left|T^{*}\right|=2$ such that $G$ contains a $T$-rigid subgraph with $k$ edges if and only if $G^{*}$ contains a $T^{*}$-rigid subgraph with $k+2(|T|-2)$ edges. This shows that the original shortest $T$-rigid subgraph problem can be reduced to the case of $|T|=2$, and hence this problem is NP-hard even when $|T|=2$.

A corollary of Theorem 1, which appears to be new (see [15]) that finding a shortest circuit in a matroid $\mathcal{M}_{2,3}(G)$ is NP-hard. To see this consider a graph $G=(V, E)$ and a designated edge $f=u v \in E$. It is known (see e.g. [9]) that if $C \subseteq E$ is a circuit of $\mathcal{M}_{2,3}(G)$ then $(V(C), C)$ is rigid. Furthermore, if $H$ is a rigid subgraph of $E-f$ then $H+f$ contains a circuit. Thus a shortest $T$-rigid subgraph of $E-f$ with respect to $T=\{u, v\}$ and $w \equiv 1$ corresponds to a shortest circuit containing $f$ in $\mathcal{M}_{2,3}(G)$.

The metric version is also hard - see [11] for the details.

Theorem 2. The metric shortest T-rigid subgraph problem is NP-hard.

In the rest of the paper we shall consider the metric version and design approximation algorithms as well as an exact algorithm (for fixed $|T|$ ).

\section{An Approximation Algorithm for the Metric Case}

Let $G=(V, E), T \subseteq V, w: E \rightarrow \mathbb{R}_{+}$be an instance of the metric shortest $T$ - $(k, k+1)$-tight subgraph problem, for some $k \geq 2$. We shall prove that the total length of a shortest $T$ - $(k, k+1)$-tight spanning subgraph of $G[T]$ is at most $(k+1) O P T$, where $O P T$ denotes the total length of an optimal solution to the shortest $T$ - $(k, k+1)$-tight subgraph problem. Since a shortest $T$ - $(k, k+1)$-tight spanning subgraph of $G[T]$ can be found in polynomial time, this leads to a $(k+1)$-approximation algorithm. In particular, we obtain a 3-approximation algorithm for the shortest $T$-rigid subgraph problem.

In our analysis we shall use the following theorem of Nash-Williams.

Theorem 3 [16]. The edge set of a graph $G=(V, E)$ can be partitioned into the edge sets of $k$ forests if and only if $i_{G}(X) \leq k|X|-k$ for all $\emptyset \neq X \subseteq V$.

A simple counting argument shows that $G[T]$ does not contain a $(k, k+1)$ tight spanning subgraph if $|T| \leq 2 k-1$ (except for $k=2$ ). Otherwise we do have a feasible solution on vertex set $T$, c.f. Lemma 2 . 
Theorem 4. Let $k$ be an integer with $k \geq 2$. Suppose that we are given a complete graph $G=(V, E)$, a terminal set $T \subseteq V$, and a metric length function $w: E \rightarrow \mathbb{R}_{+}$. If $|T| \geq 2 k$, then a shortest $T$ - $(k, k+1)$-tight spanning subgraph of $G[T]$ is a $(k+1)$-approximate solution for the metric shortest $T$ - $(k, k+1)$-tight subgraph problem in $G$.

Proof. Let $H=\left(V_{H}, E_{H}\right)$ be a shortest $T$ - $(k, k+1)$-tight subgraph of $G$. Our goal is to show that $G[T]$ contains a $T$ - $(k, k+1)$-tight subgraph whose total length is at most $(k+1) w(H)$. For simplicity we shall use $w(J)$ to denote the total length of the edges of some graph $J$.

Let $e \in\left(\begin{array}{c}V_{H} \\ 2\end{array}\right)$ be a shortest edge with both endvertices in $V_{H}$. Consider the graph $H+e$ that might have parallel edges. By Theorem 3, the edge set of $H+e$ can be partitioned into $k$ edge-disjoint spanning trees $F_{1}, \ldots, F_{k}$ on $V_{H}$. By changing the indices if necessary, we may assume that $w\left(F_{1}\right) \leq \frac{w(H)+w(e)}{k}$. Consider the graph obtained from $F_{1}$ by duplicating every edge, which is a connected Eulerian graph. Then, it contains an Eulerian walk through all vertices in $V_{H}$. Since $w$ is metric and $T \subseteq V_{H}$, by shortcutting ${ }^{1}$ this Eulerian walk, we obtain a cycle $C$ such that $V(C)=T$ and

$$
w(C) \leq 2 w\left(F_{1}\right) \leq \frac{2}{k}(w(H)+w(e)) .
$$

Let $x_{1}, \ldots, x_{t}$ be the vertices of $C$ that appear in this order along $C$, where $t=|T|$. For notational convenience, we denote $x_{t+j}=x_{j}$ for $j=1, \ldots, t$. We consider the following two cases separately.

Case 1. We first consider the case when $t=2 k$. Let $K_{2 k}$ be the complete graph with vertex set $\left\{x_{1}, \ldots, x_{2 k}\right\}$ and pick an edge $f$ in $K_{2 k}$ arbitrarily. Since the metric property implies that $w\left(x_{i} x_{i+h}\right) \leq w\left(x_{i} x_{i+1}\right)+w\left(x_{i+1} x_{i+2}\right)+\cdots+$ $w\left(x_{i+h-1} x_{i+h}\right)$ for $i \in\{1, \ldots, 2 k\}$ and for $h \in\{1, \ldots, k\}$, we have

$$
\begin{aligned}
w\left(K_{2 k}-f\right) & =\sum_{i=1}^{2 k}\left(w\left(x_{i} x_{i+1}\right)+\cdots+w\left(x_{i} x_{i+k-1}\right)\right)+\sum_{i=1}^{k} w\left(x_{i} x_{i+k}\right)-w(f) \\
& \leq \frac{k(k-1)}{2} w(C)+k(w(C)-w(e))-w(e) \\
& =\frac{k(k+1)}{2}\left(w(C)-\frac{2}{k} w(e)\right) \\
& \leq(k+1) w(H),
\end{aligned}
$$

where we use (1) in the last inequality. Furthermore, we see that $K_{2 k}-f$ is a $(k, k+1)$-tight spanning subgraph of $G[T]$ by a simple counting argument. Therefore, $G[T]$ contains a $T-(k, k+1)$-tight subgraph whose total length is at most $(k+1) w(H)$.

\footnotetext{
${ }^{1}$ We follow the walk $W$ and we shortcut every maximal subwalk that contains only non-terminal vertices and vertices already visited by $W$.
} 
Case 2. We next consider the case when $t>2 k$. Let $C_{t, k}^{\prime}$ be the $(k, k+1)$-tight subgraph of $G[T]$ defined in Lemma 2. Then, by a similar calculation to Case 1, we obtain

$$
\begin{aligned}
w\left(C_{t, k}^{\prime}\right) & =\sum_{i=1}^{2 k}\left(w\left(x_{i} x_{i+1}\right)+\cdots+w\left(x_{i} x_{i+k}\right)\right)-w\left(\left\{x_{1} x_{t}, x_{1} x_{t-1}, \ldots, x_{1} x_{t-k+1}, x_{k} x_{t}\right\}\right) \\
& \leq \frac{k(k+1)}{2} w(C)-(k+1) w(e) \\
& =\frac{k(k+1)}{2}\left(w(C)-\frac{2}{k} w(e)\right) \\
& \leq(k+1) w(H) .
\end{aligned}
$$

Therefore, $G[T]$ contains a $T$ - $(k, k+1)$-tight subgraph whose total length is at most $(k+1) w(H)$.

Since a shortest $(k, k+1)$-tight spanning subgraph of $G[T]$ can be computed by a greedy algorithm, this theorem yields a $(k+1)$-approximation algorithm for the metric shortest $T$ - $(k, k+1)$-tight subgraph problem with $|T| \geq 2 k$.

By specializing the above result to the case when $k=2$, we obtain:

Corollary 1. There is a polynomial time 3-approximation algorithm for the metric shortest T-rigid subgraph problem.

The following example shows that the approximation factor of the above algorithm is not better than 2. Suppose that every edge in $G[T]$ has length 2 , and every other edge has length 1 . Then the shortest rigid spanning subgraph of $G[T]$ has total length $4|T|-6$. On the other hand the optimum is at most $2|T|+1$ : pick two vertices $a, b \in V-T$ and consider the complete bipartite subgraph $K_{X, T}$ with color classes $X=\{a, b\}$ and $T$. By adding the edge $a b$ to this graph we obtain a feasible solution (a rigid subgraph of $G$ containing $T$ ) of total length $2|T|+1$.

Note that if $w(e) \in\{1,2\}$ for all $e \in E$ then the approximation ratio of the above algorithm is not worse than 2 . Hence, by the same example, it is equal to 2 .

Corollary 2. Let $r$ be the Steiner ratio of the metric shortest T-rigid subgraph problem. Then $\frac{1}{3} \leq r \leq \frac{1}{2}$.

\section{Optimal Solutions for Fixed $|T|$ in the Metric Case}

Consider an optimal solution $H$ to some instance of the metric shortest $T$-rigid subgraph problem. One strategy to show that the number of non-terminal vertices in $H$ is small (compared to $|T|$ ), or can be made small, is to apply specific shortcutting operations that remove vertices (or sets of vertices) of $V(H)-T$ maintaining rigidity and without increasing the total length.

This strategy works easily in the metric Steiner tree problem since degreeone vertices can be removed, degree-two vertices can be shortcut, and hence an 
upper bound on $|V(H)-T|$, in terms of $|T|$, follows immediately. This approach, with much more complicated arguments, works in the $k$-edge-connected Steiner network problem, too, see [8].

In our case $H$ is a minimally rigid graph that contains $T$. It is easy to eliminate vertices of degree-two and degree-three from $H$ (see Lemma 7 below. The number of vertices of degree at least five can be bounded by using the fact that $|E(H)|=2|V|-3$ and hence the average degree of $H$ is (a bit less than) four.

Thus the main question is whether the number of degree-four vertices in $H$ can be bounded by a function of $|T|$. We deal with this question in the next subsection.

\subsection{Reductions in Minimally Rigid Graphs}

Let $G=(V, E)$ be a minimally rigid graph and let $v \in V$ be a designated vertex with $d(v)=r$, where $d(v)$ denotes the degree of vertex $v$. The reduction operation at $v$ removes $v$ from the graph and adds $r-2$ disjoint edges connecting vertices in $N_{G}(v)$ (where $N_{G}(v)$ denotes the set of neighbours of $v$ in $G$ ). We shall be interested in the cases when $2 \leq r \leq 4$. A reduction operation is admissible if the resulting graph is also minimally rigid. We call $v$ admissible if there exists an admissible reduction at $v$. Otherwise $v$ is non-admissible.

The following lemma is well-known, see e.g. [9]. It shows that vertices of degree two and three are all admissible.

Lemma 7. Let $G=(V, E)$ be a minimally rigid graph and $v \in V$. Then

(i) if $d(v)=2$ then $G-v$ is minimally rigid,

(ii) if $d(v)=3$ then there is an admissible reduction at $v$.

Vertices of degree four may be non-admissible. In such a case there is a simple certificate of non-admissibility, as we shall prove below.

We say that $X \subseteq V$ with $|X| \geq 2$ is critical if $i_{G}(X)=2|X|-3$ holds. The next lemma is also well-known [9]. Its proof uses the fact that the function $i_{G}: 2^{V} \rightarrow Z$ is supermodular. For two disjoint sets $X, Y$ we use $d(X, Y)$ to denote the number of edges between $X$ and $Y$.

Lemma 8. Let $G=(V, E)$ be a minimally rigid graph and let $X, Y, Z$ be critical sets in $G$. Then

(i) if $|X \cap Y| \geq 2$ then $X \cap Y$ and $X \cup Y$ are also critical,

(ii) if $|X \cap Y|=1$ and $d(X-Y, Y-X) \geq 1$ then $X \cup Y$ is critical,

(iii) if $X \cap Y \cap Z=\emptyset$ and $|X \cap Y|=|X \cap Z|=|Y \cap Z|=1$ then $X \cup Y \cup Z$ is critical.

Let $v$ be a designated vertex with $d(v)=4$. We say that three critical sets $X, Y, Z \subseteq V-\{v\}$ and a vertex $p \in V-\{v\}$ form a flower $\{X, Y, Z\}$ associated with $v$, with core $p$, if

(i) $X \cap Y=X \cap Z=Y \cap Z=\{p\}$, 
(ii) $v p \in E$, and

(iii) $d(v, X-\{p\})=d(v, Y-\{p\})=d(v, Z-\{p\})=1$.

Observe that if there is a flower associated with $v$ then $v$ is non-admissible: adding a new edge connecting the core $p$ to any other neighbour of $v$ violates the sparsity condition in $V-\{v\}$.

The proofs of the next two key lemmas can be found in [11]. The first one shows that every non-admissible vertex of degree four has an associated flower.

Lemma 9. Let $G=(V, E)$ be a minimally rigid graph and let $v \in V$ be a nonadmissible vertex with $d(v)=4$. Then there exists a flower associated with $v$ in $G$.

Lemma 10. Let $G=(V, E)$ be a minimally rigid graph and let $v$ be a nonadmissible vertex of degree four. Suppose that $\{X, Y, Z\}$ form a flower associated with $v$ with core $p$. If $d(p)=4$ then $v$ and $p$ have three common neighbours.

Note that if the conditions of Lemma 10 hold then $v, p$ and their (common) neighbours induce a minimally rigid subgraph isomorphic to $K_{2,3}$ (plus the edge $p v)$.

Theorem 5. Let $G=(V, E), T \subseteq V, w: E \rightarrow Z_{+}$be an instance of the metric shortest $T$-rigid subgraph problem. Then there exists an optimal solution $H$ with $|V(H)| \leq 15|T|-1$.

Proof. Let $H=\left(V^{\prime}, E^{\prime}\right)$ be an optimal solution for which $\left|V^{\prime}\right|$ is as small as possible. We may assume that $H$ is minimally rigid. Let $S=V^{\prime}-T$ and $X=$ $\left\{v \in S: d_{H}(v)=4\right\}$. Since $w$ is metric, we can use Lemma 7 to deduce that

(i) each vertex in $S$ has degree at least four, and

(ii) each vertex in $X$ is non-admissible.

Claim. Every vertex in $X$ has at least one neighbour in $V^{\prime}-X$.

Proof. Consider a vertex $v \in X$. Since it is non-admissible, there is a flower $\{X, Y, Z\}$ with core $p$ in $H$ associated with $v$. We have $p v \in E^{\prime}$. For a contradiction suppose that $p \in X$. By Lemma 10 this implies that $v$ and $p$ have three common neighbours $x, y, z$ and the set $N_{H}(v) \cup\{v\}$ induces a minimally rigid subgraph in $H$ isomorphic to $K_{2,3}$ (plus the edge $p v$ ). It is not hard to see that $H^{\prime}:=H-\{v, p\}+\{x y, x z, y z\}$ is minimally rigid. Furthermore, $w\left(H^{\prime}\right) \leq w(H)$, since $w(x y) \leq w(x v)+w(v y), w(x z) \leq w(x p)+w(p z)$, and $w(y z) \leq w(y p)+w(p v)+w(v z)$. Thus $H^{\prime}$ is a smaller optimal solution, which contradicts the choice of $H$.

By the Claim we have

$$
d_{H}(X) \geq|X|
$$


Let $Y=S-X$, and let $T_{i}$ be the set, and $t_{i}$ be the number of vertices of degree $i$ in $T$, for $2 \leq i \leq 4$. Similarly, let $T^{+}$be the set, and $t^{+}$be the number, of vertices of degree at least five in $T$. Then we have

$$
4\left|V^{\prime}\right|-6=2\left|E^{\prime}\right|=\sum_{v \in V^{\prime}} d_{H}(v) \geq 2 t_{2}+3 t_{3}+4 t_{4}+5 t^{+}+4|X|+5|Y|,
$$

from which

$$
4\left(t_{2}+t_{3}+t^{+}+|Y|\right) \geq 2 t_{2}+3 t_{3}+5 t^{+}+5|Y|
$$

follows. Thus

$$
4 t_{2}+4 t_{3} \geq 2 t_{2}+3 t_{3}+t^{+}+|Y|
$$

and hence

$$
2|T| \geq 2\left(t_{2}+t_{3}\right) \geq 2 t_{2}+t_{3} \geq t^{+}+|Y|
$$

So we have $|Y| \leq 2|T|$. Now suppose, for a contradiction, that $\left|V^{\prime}\right| \geq 15|T|$. Since $|T \cup Y| \leq 3|T|$, we have $|X| \geq 12|T|$, and hence (2) gives

$$
d_{H}(X, T \cup Y)=d_{H}(X) \geq 12|T| .
$$

Therefore the average degree of the vertices in $T \cup Y$ is at least four in $H$. This implies

$$
4\left|V^{\prime}\right|-6=2\left|E^{\prime}\right|=\sum_{v \in X} d_{H}(v)+\sum_{v \in T \cup Y} d_{H}(v) \geq 4|X|+4\left|V^{\prime}-X\right|=4\left|V^{\prime}\right|,
$$

a contradiction. Hence $\left|V^{\prime}\right| \leq 15|T|-1$, completing the proof of the theorem.

We can use this result to argue that if we compute a shortest rigid subgraph with vertex set $V^{\prime}$ for every $V^{\prime} \subseteq V$ with $T \subseteq V^{\prime}$ and $\left|V^{\prime}\right| \leq 15|T|-1$, the shortest one will correspond to an optimal solution to the shortest $T$-rigid subgraph problem. Since we can find a shortest rigid subgraph on $V^{\prime}$ in polynomial time for each $V^{\prime}$, we obtain:

Theorem 6. The metric shortest T-rigid subgraph problem can be solved in polynomial time for fixed $|T|$.

\section{Concluding Remarks}

The Steiner problem for count matroids, introduced in this paper, gives rise to numerous open problems. The most obvious ones are about potential improvements of the new results: better approximation factors, better bounds for the Steiner ratio, and extensions to further parameters $(k, l)$.

Two Terminals. For the complexity status of the two-terminal case (with general length functions) there seems to be a clean answer. We conjecture that the proof of Theorem 1 can be extended to all count parameters $(k, l)$ with $k \geq 2$. The remaining cases (assuming $l \geq 0$ ) are $(1,1)$ and $(1,0)$. The former case corresponds to the familiar shortest path problem, which is polynomial time 
solvable by using Dijkstra's algorithm. The latter case is also tractable. Recall that the count matroid $\mathcal{M}_{1,0}$ is the so-called bicircular matroid, in which a graph $H$ is tight if and only if each connected component of $H$ is unicyclic, that is, it has exactly one cycle.

Theorem 7. The shortest (1,0)-tight subgraph problem with $|T|=2$ is polynomial time solvable.

The proof of this results as well as further comments and potential research directions are given in [11].

\section{References}

1. Berg, A.R., Jordán, T.: Algorithms for graph rigidity and scene analysis. In: Di Battista, G., Zwick, U. (eds.) ESA 2003. LNCS, vol. 2832, pp. 78-89. Springer, Heidelberg (2003). https://doi.org/10.1007/978-3-540-39658-1_10

2. Byrka, J., Grandoni, F., Rothvoss, T., Sanita, L.: An improved LP-based approximation ratio for Steiner tree. In: Proceedings of 42nd STOC, pp. 583-592 (2010)

3. Chung, F.R.K., Graham, R.L.: A new bound for Euclidean Steiner minimal trees. Ann. New York Acad. Sci. 440, 328-346 (1985)

4. Du, D., Hu, X.: Steiner Tree Problems in Computer Communication Networks. World Scientific Publishing, River Edge (2008)

5. Frank, A.: Connections in Combinatorial Optimization. Oxford University Press, Oxford (2011)

6. Frank, A., Szegő, L.: Constructive characterizations for packing and covering with trees. Discrete Appl. Math. 131, 347-371 (2003)

7. Jackson, B., Jordán, T.: Graph theoretic techniques in the analysis of uniquely localizable sensor networks. In: Mao, G., Fidan, B. (eds.) Localization Algorithms and Strategies for Wireless Sensor Networks, pp. 146-173. IGI Global, Hershey (2009)

8. Jordán, T.: On minimally $k$-edge-connected graphs and shortest $k$-edge-connected Steiner networks. Discrete Appl. Math. 131, 421-432 (2003)

9. Jordán, T.: Combinatorial rigidity: graphs and matroids in the theory of rigid frameworks. Discrete Geom. Anal. MSJ Memoirs 34, 33-112 (2016)

10. Jordán, T., Domokos, G., Tóth, K.: Geometric sensitivity of rigid graphs. SIAM J. Discrete Math 27(4), 1710-1726 (2013)

11. Jordán, T., Kobayashi, Y., Mahara, R., Makino, K.: The Steiner problem for count matroids, TR-2020-03, Egerváry Research Group, Budapest

12. Laman, G.: On graphs and rigidity of plane skeletal structures. J. Eng. Math. 4, 331-340 (1970)

13. Lee, A., Streinu, I.: Pebble game algorithms and sparse graphs. Disc. Math 308, $1425-1437$ (2008)

14. Lomonosov, A.: Graph and combinatorial algorithms for geometric constraint solving, Ph.D. thesis, U. Florida (2004)

15. Matroids - girth and co-girth. In: Egres Open, an open problem collection of the Egerváry Research Group, Budapest. lemon.cs.elte.hu/egres/open

16. Nash-Williams, C.St.J.A.: Decomposition of finite graphs into forests. J. Lond. Math. Soc. 39, 12 (1964)

17. Whiteley, W.: Some matroids from discrete applied geometry. Contemp. Math. 197, 171-311 (1996). In: Matroid theory (Seattle, WA, 1995), Amer. Math. Soc., Providence, RI 